\title{
In-hospital mortality from Coronavirus Disease 2019 in a northern Italian centre:results of a competing risk analysis from SMAtteo COvid19 REgistry (SMACORE)
}

Valentina Zuccaro

IRCCS Fondazione San Matteo

Ciro Celsa

University of Palermo

Margherita Sambo

IRCCS Fondazione San Matteo

Salvatore Battaglia

University of Palermo

Paolo Sacchi

IRCCS Fondazione San Matteo

Simona Biscarini

IRCCS Fondazione San Matteo

Pietro Valsecchi

IRCCS Fondazione San Matteo

Teresa Chiara Pieri

IRCCS Fondazione San Matteo

Ilaria Gallazzi

IRCCS Fondazione San Matteo

Marta Colaneri

IRCCS Fondazione San Matteo

Michele Sachs

IRCCS Fondazione San Matteo

Silvia Roda

IRCCS Fondazione San Matteo

Erika Asperges

IRCCS Fondazione San Matteo

Matteo Lupi

IRCCS Fondazione San Matteo

Alessandro Di Filippo

IRCCS Fondazione San Matteo

Elena Seminari

IRCCS Fondazione San Matteo 


\section{Angela Di Matteo}

IRCCS Fondazione San Matteo

\section{Stefano Novati}

IRCCS Fondazione San Matteo

\section{Laura Maiocchi}

IRCCS Fondazione San Matteo

\section{Marco Enea}

University of Palermo

Massimo Attanasio

University of Palermo

\section{Calogero Cammà}

University of Palermo

Raffaele Bruno ( $\nabla$ raffaele.bruno@unipv.it)

IRCCS Fondazione San Matteo, University of Pavia

\section{Research Article}

Keywords: COVID-19, SARS-CoV-2, in-hospital death, intensive care, hospital discharge

Posted Date: July 16th, 2020

DOI: https://doi.org/10.21203/rs.3.rs-43352/v1

License: (c) (i) This work is licensed under a Creative Commons Attribution 4.0 International License. Read Full License 


\section{Abstract}

Objectives An accurate prediction of the clinical outcomes of European patients requiring hospitalisation for Coronavirus Disease 2019 (COVID-19) is lacking. The aim of the study is to identify predictors of in-hospital mortality and discharge in a cohort of Lombardy patients with COVID-19.

MethodsAll consecutive hospitalised patients from February $21^{\text {st }}$ to March $30^{\text {th }}, 2020$, with confirmed COVID19 from the IRCCS Policlinico San Matteo, Pavia, Lombardy, Italy, were included. In-hospital mortality and discharge were evaluated by competing risk analysis. The Fine and Gray model was fitted in order to estimate the effect of covariates on the cumulative incidence functions (CIFs) for in-hospital mortality and discharge.

Results 426 adult patients (median age 68 (IQR, 56 to 77 years) were admitted with confirmed COVID-19 over a 5-week period; 292 (69\%) were male. By 21 April 2020, 141 (33\%) of these patients had died, 239 (56\%) patients had been discharged and $46(11 \%)$ were still hospitalised. Regression on the CIFs for in-hospital mortality showed that older age, male sex, number of comorbidities and hospital admission after March $4^{\text {th }}$ were independent risk factors associated with in-hospital mortality.

Conclusions Olderage, male sex and number of comorbidities definitively predicted in-hospital mortality in hospitalised patients with COVID-19

\section{Introduction}

Since December 2019 SARS COV 2 disease, defined as a pandemic by the World Health Organization (WHO) on 11 March 2020, has spread rapidly all over the world. ${ }^{1}$ Outside China, the first western country to be affected was Italy, where the epidemic began on 21 February 2020 and quickly affected thousands of people, practically overwhelming the capacity of the National Health System to respond to it in terms of availability of hospital, ICU beds and ER spaces to receive and manage patients. ${ }^{2}$ One of the first factors limiting the ability to best manage COVID-19 patients was the difficulty in allocating them, because clinical criteria to define the evolution of the disease were, and still are, missing.

To date, most of the studies that have extensively reported the clinical and laboratory characteristics of patients infected by COVID-19 have been carried out in China. ${ }^{3}$ Data on clinical outcomes and treatment of COVID- 19 outside China are lacking and the high heterogeneity in observed case-fatality ratios between and within different countries still remains unexplained. Because COVID-19 shows an array of clinical presentations and the lack of effective treatment makes it difficult to predict its outcome, the identification of risk factors for clinical outcomes, such as death, ICU admission and hospital discharge is crucial in order to improve the organisation of healthcare and to identify patients who may benefit the most from the available treatment strategies. Moreover, in such a complex epidemiological and clinical scenario, competing risks might help in the assessment of the impact of treatment strategies on meaningful clinical endpoints, such as in-hospital death and discharge. ${ }^{4}$ The aim of this study was to explore and explain, in a cohort of Lombardy patients with COVID-19 in Pavia, Italy, the heterogeneity of clinical outcomes and to identify predictors of inhospital mortality and discharge by competing risks analysis. 


\section{Results}

From 22 February to 30 March 2020, 426 confirmed cases of COVID-19 were observed, 292 (68.5\%) were males (Table 1). Median age was 68 years (IQR, 56 to 77 years) and 197 (45.8\%) patients were older than 70 years of age. 269 (63\%) patients had at least one comorbidity, with hypertension and diabetes being the most common (140 (33\%) and $63(15 \%)$ patients, respectively). The median score on the Charlson comorbidity index was 3 (IQR, 1 to 4). The first nasal swab test for SARS-COV2 was positive in 365 (86\%) patients, while $61(14 \%)$ patients had a negative first nasal swab test and positive repeat nasal swab test.

Laboratory findings on admission are reported in Table 1. Lymphocytopenia was present in 398 (93.3\%) patients, while platelet count was lower than $150,000 / \mathrm{mmc}$ in $100(23.5 \%)$ patients. CRP was increased in $188(44.0 \%)$ patients and LDH was elevated in 369 (87.0\%) patients. Chest radiography revealed the presence of interstitial pneumonia in $301(71.0 \%)$ patients.

Data on treatments are reported in Table 2. Antibiotic therapy was started in 304 (85\%) of patients. Corticosteroid treatment was administered to $70(20 \%)$ patients and consisted of dexamethasone $20 \mathrm{mg}$ daily in 13 patients and, starting on 21 March 2020, methylprednisolone $1 \mathrm{mg} / \mathrm{kg}$ intravenously daily in 57 patients. Hydroxycloroquine, $600 \mathrm{mg}$ twice on day 1, then $400 \mathrm{mg}$ daily for 7 days, was administered to 249 (70.3\%) patients and was initiated within 72 hours following admission. 64 (18.1\%) patients did not receive any antiviral drug, while 174 (49.1\%) patients received antiviral treatment with Lopinavir/ritonavir 400/100 mg twice daily. 22 (5.2\%) patients received Tocilizumab 8 mg/kg from 13 March 2020.

\section{Clinical outcomes}

On 21 April 2020, 141 (33.1\%) patients had died. The median time from symptoms onset to death and from hospitalisation to death was 11 days (IQR 3-19) and 6 days (IQR 3-11), respectively. 41 (9.6\%) patients had been transferred to ICU. The median time from hospitalisation to ICU admission was 4 days (IQR 2-6). 239 (56\%) patients had been discharged and $46(10.7 \%)$ patients were still hospitalised (17 of whom were still in ICU). Median time from hospitalisation to discharge was 10 days (IQR 5-18).

Patients who died were older, had higher Charlson comorbidity index, higher CRP and LDH levels and lower lymphocyte count compared to survivor patients (Table 1).. Hydroxycloroquine and antibiotics were used more frequently in patients who died compared to those who did not. The frequency of complications, such as respiratory failure, acute kidney injury, acute cardiac injury and septic shock was significantly higher in patients who died as compared to survivors. (Table S1).

The CIF for in-hospital mortality is showed in Figure 1. The estimated probability of in-hospital death was $24.4 \%$ during the first 10 days from hospitalization, $31.0 \%$ during the first 20 days and $33.7 \%$ at the end of follow-up. Univariate analysis for in-hospital mortality is reported in Table S2. Using the Fine and Gray modelto-model mortality, older age ( $70-79$ years: HR $4.42,95 \% \mathrm{Cl} 2.59-7.39, \mathrm{p}<0.0001$. Over 79 years: HR 7.75, $95 \% \mathrm{Cl} 4.39-13.74, \mathrm{p}<0.0001)$, male sex (HR 1.85, 95\% Cl 1.22-2.89, $\mathrm{p}=0.003)$, number of comorbidities higher than 3 (HR 3.63, $p=0.03$ ), and time of hospital admission (between 4 March and 16 March: HR 2.32, $95 \% \mathrm{Cl} 1.45-3.71, \mathrm{p}=0.001$; between 17 March and 30 March: HR 1.68, 95\% Cl 1.03-2.75, $\mathrm{p}=0.04$ ) were 
independently associated with higher in-hospital mortality, while time to ICU admission longer than 7 days (HR $0.19,95 \% \mathrm{Cl} 0.05-0.67, \mathrm{p}=0.01$ ) were independently associated with lower in-hospital mortality (Table 3). The CIFs for in-hospital mortality performed using the parameter estimates of the Fine and Gray model for each of these covariates are showed in Figures S1-S4.

These risk factors were then used to construct a model encompassing all patients grouped into a "best" and a "worst" class according to the presence or not of these factors. CIFs for the best class (female patients with less than 3 comorbidities, admitted between February, 21 and March, 3) and for the worst class (male patients with more than 3 comorbidities, hospitalized between 4 March and 16 March) stratified by age group are showed in Figure 2. At the end of follow-up, the probability of in-hospital death in patients younger than 70 years was $1.8 \%$ in the best class and $18.6 \%$ in the worst class. In patients with $70-79$ years, the probability of in-hospital death at the end of follow-up was $8.3 \%$ in the best class and $62.5 \%$ in the worst class. In patients older than 80 years, the probability of in-hospital death at the end of follow-up was $13.7 \%$ in the best class and $80.8 \%$ in the worst class.

The characteristics and outcomes of patients according to the discharge status are reported in Tables S3-S4. The CIF for discharge is showed in Figure 1. The estimated probability of discharge was $30.5 \%$ during the first 10 days from hospitalization, $48.8 \%$ during the first 20 days and $61.4 \%$ at the end of follow-up Univariate analysis is reported in Table S2. Tocilizumab use was significantly associated with a lower probability to be discharged at univariate analysis, however it was not included in the multivariate model because only 22 patients received Tocilizumab. Using the Fine and Gray model, we observed that lymphocytes count (HR $1.13,95 \% \mathrm{Cl} 1.06-1.19, \mathrm{p}=0.0001)$ was independently associated with higher probability to be discharged, while older age ( $70-79$ years: HR $0.39,95 \% \mathrm{Cl} 0.27-0.55$, $\mathrm{p}<0.0001$. Over 79 years: HR $0.27,95 \% \mathrm{Cl} 0.16-$ $0.44 p<0.0001)$, number of comorbidities higher than 3 (HR 0.08, $p<0.0001)$, and time of hospital admission (between March, 4 and March, 16: HR 0.66, 95\% Cl 0.47-0.92, p = 0.02; between 17 March and 30 March: HR $0.68,95 \% \mathrm{Cl} 0.50-0.93, \mathrm{p}=0.02$ ) were independently associated with lower probability to be discharged. (Table 3). The CIFs for discharge performed using the parameter estimates of the Fine and Gray model for each of these covariates are showed in Figures S5-S9. The CIFs for the best class and for the worst class according to age are showed in Figures S10-S12. At the end of follow- up, the probability of discharge in patients younger than 70 years was $99.5 \%$ in the best class and $31.5 \%$ in the worst class. In patients with 70-79 years, the probability of discharge at the end of follow-up was $84.6 \%$ in the best class and $12.6 \%$ in the worst class. In patients older than 80 years, the probability of discharge at the end of follow-up was $75.3 \%$ in the best class and $9.6 \%$ in the worst class.

\section{Discussion}

This report, to our knowledge, is the first large retrospective study of consecutive hospitalised patients with confirmed COVID-19 in Europe. Thus far, descriptions of retrospective data of cohorts of COVID-19 patients show results which are, for the most part, limited by some biases, such as the heterogeneity of subjects enrolled, as well as that of medical interventions. Therefore, the interpretation of data should move from an analysis of the population and progress to describing the kind of interventions carried out and the outcomes 
obtained. It would be significant to compare them with controls; however, this possibility is currently nonexistent due to the pandemic emergency.

The median age in our cohort was 68 years and 77 years in patients who died, which is higher than that observed in other studies. In-hospital mortality assessed by competing risks analysis was significantly higher in patients aged between 70 and 79 years and in those over 79, compared with patients younger than 70 years. By contrast, the probability of discharge was similar between patients of 70-79 years and those older than 79 years. Hypertension, diabetes and other cardiovascular comorbidities, such as coronary heart disease and atrial fibrillation, were the most common. Notably, the median score on the Charlson comorbidity index was 3 in our cohort, which corresponds to about an $80 \%$ estimated 10 -year survival, reflecting a significant comorbidity burden 4 . Our results are in line with those of the Italian National Institute of Health, showing that approximately $61 \%$ of deceased Italian patients with COVID-19 had more than 3 comorbidities, while only $3.6 \%$ of patients who died had no comorbidity. ${ }^{5}$ Male sex was an independent risk factor for inhospital mortality and a lower probability of discharge.

When comparing our cohort with those described in the literature we noted that mortality was higher than that observed in other studies conducted both in and outside China. ${ }^{6-8}$ While the prevalence of comorbidities in our cohort was similar to that reported in the USA, ${ }^{7}$ it was higher than that observed in Chinese cohorts. ${ }^{6,8}$ The association between age and in-hospital mortality could be explained by the lower cardiopulmonary reserve, by the enhanced susceptibility to infections and by the inadequate control of anti-inflammatory mechanisms. ${ }^{9}$ The association between gender and worst outcomes in COVID-19 is not fully understood. It has been proposed that female sex could be associated with a lower susceptibility to viral infections, with sex hormones playing a relevant role in innate and adaptive immune response. ${ }^{10} \mathrm{~A}$ different expression of ACE 2 receptor has also been suggested as an explanation of the gender-associated mortality in COVID-19 patients. ${ }^{11}$ Conversely, it has been suggested that males could be more prone to being affected by COVID19 due to the higher smoking rate and higher prevalence of cardiovascular comorbidities. ${ }^{12}$ However, our multivariate model suggested that sex was an independent predictor of mortality, and discharge regardless of comorbidities and evidence supporting smoking as a predisposing factor in men with COVID-19 are lacking. Unfortunately, we were unable to evaluate the association between smoking and clinical outcomes in COVID-19.

In-hospital mortality was high (33\%), and patients who were admitted during the first weeks of the emergency had a significantly lower in-hospital mortality and a higher likelihood of discharge compared to those who were admitted during subsequent weeks, with the worst outcomes observed from 4 March to 16 March 2020. One factor that many reports have addressed is the sequence of phases into which the disease has been divided, each corresponding to a different pattern of viral and immunological factors. Patient presentation in late phase may also have occurred, leading to the admission of an exceptionally large number of patients who needed hospitalisation in a short time span, resulting in a critical overload in the Policlinico San Matteo, in both triage and the management of the disease. These findings may be explained by also taking into consideration that during the first week many admissions were made for epidemiological reasons, leading to the hospitalisation of patients with few symptoms or mild disease. 
Notably, no antiviral treatment was found to be associated with any improvement in mortality and discharge. Regarding lopinavir/ritonavir in particular, our findings confirm, in a European cohort collected in a real- world setting, the results of a recent randomised controlled trial that did not show any benefits from lopinavir/ritonavir treatment beyond standard care in a Chinese population. ${ }^{13}$ Similarly, we did not observe any significant differences in the in-hospital mortality between patients exposed or unexposed to hydroxichloroquine. Although Tocilizumab was significantly associated with a lower probability of discharge at univariate analysis, the small sample size of treated patients and potential selection bias of physicians to give anticytokine agents to the most severe patients hampered robust conclusions regarding this drug. Although ICU admission after 7 days from hospitalisation was independently and significantly associated with a lower risk of in-hospital mortality, the rapidity with which patients entered the ICU often concurrently with initiating other treatments, making the benefit of this treatment difficult to assess. Moreover, results from observational studies of drug effects should be interpreted with caution as they may be biased by survivor treatment selection bias, including time-related biases. ${ }^{14,15}$

In the literature, the use of composite endpoints (i.e. death or ICU admission) and, on the other hand, the implementation of traditional survival and Cox models are not appropriate in a disaster medicine setting such as that of COVID-19. The first assumption considers ICU and death to be equal, which is not true, while the traditional Cox model neglects to model discharge as an alternative endpoint. Competing risks analysis may provide further insights into the effect of interventions on the separate endpoint components. ${ }^{16} \mathrm{We}$ overcame this issue by performing a competing risks analysis taking into account two events (in-hospital death and discharge) and including ICU admission as a time-dependent covariate. ${ }^{17}$ We suggest the use of a standardised methodology to assess treatment effects in observational studies in the complex clinical scenario of COVID-19. Summarising all the available evidence from randomised controlled trials and realworld comparative effectiveness studies, we are convinced that effective treatments for COVID-19 are still lacking and that therapies, such as specific antiviral drugs and immunomodulatory agents, remain an unmet and urgent medical need.

The main limitation of our study is the retrospective design. Retrospective studies have many problems that reduce their internal and external validity. When assessing retrospective cohort studies, the most important bias is the likelihood of the inappropriate selection of patients, which can lead to incorrect results and spurious associations. However, we included only consecutive patients with confirmed COVID-19, therefore we believe that selection bias was not relevant. Moreover, some potential confounders associated with the severity of COVID-19 (i.e. P/F ratio or circulating cytokine levels) and not available for this modelling could affect our results. Thus, we performed multivariate competing risks analysis to overcome this issue. Other limitations are the generalisability of our results to different populations and settings, particularly regarding the demographic structure of our country, including European elderly patients with a high prevalence of comorbidities. Finally, mortality was limited to in-hospital death, and discharged patients were assumed to still be alive during the study period.

In conclusion, our findings indicate that in a Lombardy cohort of elderly hospitalized patients, for the most part male with a high prevalence of comorbidities, COVID-19 is characterised by high in-hospital mortality. 
Older age, male sex, comorbidities and time of admission were found to be significant risk factors for inhospital mortality and associated with a lower probability of being discharged.

\section{Methods}

\section{Study setting}

The SMatteo COvid19 Registry (SMACORE) is a cohort of patients with a confirmed diagnosis of COVID- 19 disease referred to the IRCCS Policlinico San Matteo Hospital of Pavia, Italy from February 2020. The SMACORE database includes demographic, clinical laboratory tests, treatment, and outcome data. Ethics approval for observational research using SMACORE data was obtained from the local ethics committee.

This is a single centre, retrospective, observational cohort study and all patients of SMACORE cohort consecutively admitted to the Infectious Diseases Unit between 22 February and 30 March 2020, with a diagnosis of COVID-19 were enrolled. ICD-9 CM codes were reviewed, and clinical data were extracted. Patients were followed until 21 April 21 2020. Laboratory confirmation of the SARS COV-2 infection was defined as positive Real-Time Reverse Transcriptase Polymerase Chain Reaction (RT-PCR) from clinical nasal swab.

\section{Statement}

All methods were carried out in accordance with relevant guidelines and regulations and ethics approval for observational research using SMACORE data was obtained from the local ethics committee and the informed consent has been obtained as by internal procedures.

\section{Data source}

Demographic, clinical, laboratory, treatment, and outcome data were extracted from medical records using a standardised data collection form. The Charlson comorbidity index was used to assess comorbidity. It includes 16 comorbidities, predicting 10-year survival in patients with multiple comorbidities and was used as a measure of the total comorbidity burden. ${ }^{18}$ Imaging examinations were based on chest X-ray results.

Although the benefits of a chest CT scan in achieving an early diagnosis of COVID-19 and in the follow-up of pneumonia evolution are well known, ${ }^{19}$ we did not have the opportunity to include them in our clinical workout.

\section{Laboratory tests}

Respiratory samples from the upper respiratory tract were prospectively collected and analysed at the Molecular Virology Unit, Fondazione IRCCS Policlinico San Matteo, Pavia, Italy, as part of the Regional SARSCoV-2 surveillance and diagnosis plan in the Lombardy region. Total nucleic acids (DNA/RNA) were extracted from 200 ul of UTM ${ }^{\mathrm{TM}}$ using the QIAsymphon ${ }^{\circledR}$ instrument with QIAsymphony ${ }^{\circledR}$ DSP 
Virus/Pathogen Midi Kit (Complex 400 protocol) according to the manufacturer's instructions (QIAGEN, Qiagen, Hilden, Germany). Specific RT-PCR targeting RNA-dependent RNA polymerase and E genes were used to detect the presence of SARS-CoV-2 in respiratory samples according to the WHO guidelines and published protocols. ${ }^{20,21}$

Routine blood examinations included complete blood count, serum creatinine, glutamic oxaloacetic transaminase (GOT) and glutamic pyruvic transaminase (GPT), lactate dehydrogenase (LDH), C-reactive protein (CRP) and troponin. Lymphocitopenia was defined as lymphocyte count $<1.5 \times 10^{9} / \mathrm{L}$. CRP was considered elevated above $10 \mathrm{mg} / \mathrm{dL}$. LDH levels were considered elevated above $245 \mathrm{U} / \mathrm{L}$. Blood cultures were performed in each patient and arterial-blood gas analysis (ABG) was performed when clinical signs of oxygen impairment were detected (e.g. tachypnoea and hypoxia).

\section{Treatment data}

Treatment data included use of lopinavir/ritonavir, hydroxychloroquine, corticosteroids, tocilizumab and antibiotic drugs. Lopinavir/ritonavir 400/100 mg was administered orally twice daily for 14 days.

Hydroxychloroquine (HCQ) 600 mg twice on day 1, then 400 mg daily for 7 days. Corticosteroid treatment consisted of dexamethasone $20 \mathrm{mg}$ daily for 5 days in patients admitted from 22 February to 20 March and methylprednisolone $1 \mathrm{mg} / \mathrm{kg}$ intravenously daily for 5 days from 21 March to the end of follow-up.

Tocilizumab $8 \mathrm{mg} / \mathrm{kg}$ was given intravenously in 1 or 2 doses from 13 March to the end of follow-up. A second dose was given 8-12 hours after the first dose in patients with inadequate response. Antibiotic therapy consisted of a combination of piperacillin/tazobactam and doxycycline. Low (cannula and simple masks) and high (Venturi and reservoir masks, Nasal High Flow (NHF), helmet continuous positive airway pressure (CPAP)) flow oxygen support was provided when hypoxia was detected. Time to ICU admission was defined as the time from hospitalisation to ICU admission.

\section{Outcomes}

The primary disease event was in-hospital mortality. Discharge was analysed as a competing event by competing risks analysis.

The criteria for discharge were absence of fever, clinical remission of respiratory symptoms, oxygen saturation greater than $94 \%$ and two nasal swab samples negative for SARS-CoV-2 RNA obtained at least 24 hours apart.

Septic shock was defined according to the 2016 Third International Consensus Definition for Sepsis and Septic Shock. ${ }^{22}$ Acute kidney injury was defined according to the KDIGO clinical practice guidelines ${ }^{23}$ and acute cardiac injury was diagnosed if serum levels of cardiac biomarkers (troponin) was above the $99^{\text {th }}$ percentile upper reference limit, or if new abnormalities were shown in electrocardiography and echocardiography. ${ }^{24}$ 


\section{Statistical analysis}

Data for continuous variables are presented as mean and standard deviation or median and interquartile ranges (IQR), and data for categorical variables are presented as frequency and percentage. Differences between continuous data were analysed by Student $t$ test or by Mann-Whitney $U$ test. Differences between categorical variables were analysed by $\chi^{2}$ test.

In-hospital mortality and discharge were evaluated by competing risks analysis, using cumulative incidence function (CIF). ${ }^{4}$ The proportional sub-distribution hazard model by Fine and Gray was fitted in order to estimate the effect of covariates on CIFs in-hospital death and discharge, including ICU admission as a timedependent covariate. ${ }^{25}$ Covariates used for multivariate analyses were chosen based on their significance in the univariate analysis $(p<0.10)$. Variables in the final model with a $p$-value $<0.05$ were considered statistically significant. The results are expressed as adjusted hazard ratios (HR) and their $95 \%$ confidence intervals $(\mathrm{Cl})$. Models used a complete-case analysis approach. Statistical analyses were completed in SAS version 9.4 .

\section{Declarations}

\section{Contributor and guarantor information}

The corresponding author attests that all the listed authors meet authorship criteria and that no others meeting the criteria have been omitted. VZ and CC contributed equally to the manuscript. VZ, CC, RB, and CC conceived the study. MS, PS, SB, PV, TCP, IG, MC, MS, SR, EA, ML, AD, ES, AD, SF, and LM collected the data and provided comments and feedback for the manuscript. SB, ME, and MA coded the methods and performed the statistical analysis. CC, CC, VZ, and RB wrote the manuscript and provided comments and feedback. All authors read and approved the final version of the manuscript. RB acts as guarantor.

Competing interests: All authors have completed the ICMJE uniform disclosure form at www.icmje.org/coi_disclosure.pdf and declare: no support from any organisation for the submitted work; no financial relationships with any organisations that might have an interest in the submitted work in the previous three years; no other relationships or activities that could appear to have influenced the submitted work.

Ethics Approval The study was approved by Fondazione IRCCS Policlinico San Matteo institutional review board for observational research using SMACORE data.

Data sharing The authors agree to share relevant, anonymized data generated as part of the SMAtteo COvid19 REgistry (SMACORE) upon reasonable request.

Transparency statement The manuscript's guarantor (RB) affirms that the manuscript is an honest, accurate, and transparent account of the study being reported; that no important aspects of the study have been omitted; and that any discrepancies from the study as originally planned have been explained. 
Role of the funding source There are no sources of funding to disclose. All authors, external and internal, had full access to all of the data (including statistical reports and tables) in the study and can take responsibility for the integrity of the data and the accuracy of the data analysis.

\section{References}

1. https://who.int/dg/speeches/detail/who-director-general-s-opening-remarks-at-the-media- briefing-oncovid-19 11-march-2020

2. Remuzzi A, Remuzzi COVID-19 and Italy: what next? Lancet. 2020;395(10231):1225-1228.

3. Rodriguez-Morales AJ, Cardona-Ospina JA, Gutiérrez-Ocampo E, et al. Clinical, laboratory and imaging features of COVID-19: A systematic review and meta-analysis [published online ahead of print, 2020 Mar 13]. Travel Med Infect Dis. 2020;34:101623.

4. Putter H, Fiocco M, Geskus RB. Tutorial in biostatistics: competing risks and multi-state Stat Med. 2007;26(11):2389-2430.

5. Istituto superiore di Sanità. Characteristics of COVID-19 patients dying in Italy. Report based on available data on May 7th, 2020. https://epicentro.iss.it/en/coronavirus/sars-cov-2-analysis-of- deaths

6. Zhou F, Yu T, Du R, et al. Clinical course and risk factors for mortality of adult inpatients with COVID-19 in Wuhan, China: a retrospective cohort study. Lancet. 2020 Mar 28;395(10229):1054- 1062.

7. Richardson S, Hirsch JS, Narasimhan M, et al. Presenting Characteristics, Comorbidities, and Outcomes Among 5700 Patients Hospitalized With COVID-19 in the New York City Area [published online ahead of print, 2020 Apr 22]. JAMA. 2020;e206775. doi:10.1001/jama.2020.6775.

8. Wang D, Hu B, Hu C, et al. Clinical Characteristics of 138 Hospitalized Patients With 2019 Novel Coronavirus-Infected Pneumonia in Wuhan, China [published online ahead of print, 2020 Feb 7]. JAMA. 2020;323(11):1061- doi:10.1001/jama.2020.1585

9. Opal SM, Girard TD, Ely EW. The immunopathogenesis of sepsis in elderly patients. Clin Infect Dis 2005; 41 (suppl 7): S504-12.

10. Jaillon S, Berthenet K, Garlanda C. Sexual Dimorphism in Innate Immunity. Clin Rev Allergy Immunol. 2019 Jun;56(3):308-321.

11. Cao Y, Li L, Feng Z,Wan S, Huang P, Sun X,Wen F, Huang X, Ning G, Wang W. Comparative genetic analysis of the novel coronavirus (2019-nCoV/SARS-CoV-2) receptor ACE2 in different populations.

12. Cai H. Sex difference and smoking predisposition in patients with COVID-19 [published correction appears in Lancet Respir Med. 2020 Apr;8(4):

13. Cao B, Wang Y, Wen D, et al. A Trial of Lopinavir-Ritonavir in Adults Hospitalized with Severe Covid-19. N Engl J Med. 2020;382(19):1787-

14. Suissa S, Azoulay Metformin and the risk of cancer: time-related biases in observational studies. Diab Care 2012;35:2665-2673.

15. Glesby MJ, Hoover DR. Survivor treatment selection bias in observational studies: examples from the AIDS literature. Ann Intern Med 1996;124:999-1005. 
16. Wolbers M, Koller MT, Stel VS, et al. Competing risks analyses: objectives and approaches. Eur Heart J. 2014;35(42):2936- doi:10.1093/eurheartj/ehu131

17. Putter $\mathrm{H}$, Schumacher $\mathrm{M}$, van Houwelingen HC. On the relation between the cause-specific hazard and the subdistribution rate for competing risks data: The Fine-Gray model revisited. Biom J. 2020;62(3):790doi:10.1002/bimj.201800274

18. Charlson ME, Pompei P, Ales KL, MacKenzie CR. A new method of classifying prognostic comorbidity in longitudinal studies: development and validation. J Chronic Dis. 1987;40(5):373-383.

19. 6 ex Shi H, Han X, Jiang N, et al. Radiological findings from 81 patients with COVID-19 pneumonia in Wuhan, China: a descriptive study. Lancet Infect Dis. 2020;20(4):425-434.

20. Coronavirus disease (COVID-19) technical guidance: Laboratory testing for 2019-nCoV in humans. https://who.int/emergencies/diseases/novel-coronavirus-2019/technical-guidance/laboratory-guidance

21. Corman VM, Landt O, Kaiser M, et al. Detection of 2019 novel coronavirus (2019-nCoV) by real- time RTPCR. Euro Surveill. 2020;25(3):2000045.

22. Singer M, Deutschman CS, Seymour CW, et al. The Third International Consensus Definitions for Sepsis and Septic Shock (Sepsis-3). JAMA 2016; 315: 801-10.

23. Kidney Disease: Improving Global Outcomes (KDIGO) Acute Kidney InjuryWork Group. KDIGO Clinical Practice Guideline for Acute Kidney Injury. Kidney Int Suppl. 2012;2:1.

24. Gao C, Wang Y, Gu X, et al. Association Between Cardiac Injury and Mortality in Hospitalized Patients Infected With Avian Influenza A (H7N9) Virus. Crit Care Med. 2020;48(4):451-

25. Fine JP et al. A proportional hazards model for the sub-distribution of a competing risk. J Am Stat Assoc 1999; 94:496-509.

\section{Tables}

Table 1. Demographic, clinical and laboratory characteristic of patients on admission. 


\begin{tabular}{|c|c|c|c|c|}
\hline & Overall $(n=426)$ & Death $(n=141)$ & Survivor $(\mathrm{n}=285)$ & $p$-value \\
\hline Age (years) & $68.0(56.0-77.0)$ & $77.0(71.0-83.0)$ & $61.0(50.0-72.0)$ & $<0.0001$ \\
\hline$<50$ & $72(16.9 \%)$ & $1(0.7 \%)$ & $71(24.9 \%)$ & $<0.0001$ \\
\hline $50-59$ & $64(15.0 \%)$ & $8(5.7 \%)$ & $56(19.6 \%)$ & 0.0003 \\
\hline $60-69$ & $95(22.3 \%)$ & $21(14.9 \%)$ & $74(25.9 \%)$ & 0.014 \\
\hline 70-79 & $125(29.3 \%)$ & $66(46.8 \%)$ & $59(20.7 \%)$ & $<0.0001$ \\
\hline$>80$ & $70(16.4 \%)$ & $45(31.9 \%)$ & $25(8.8 \%)$ & $<0.0001$ \\
\hline Male sex & $292(68.5 \%)$ & $103(73.0 \%)$ & $189(66.3 \%)$ & 0.194 \\
\hline Comorbidity & $269(63.1 \%)$ & $116(82.2 \%)$ & $153(53.7 \%)$ & $<0.0001$ \\
\hline Hypertension & $140(32.8 \%)$ & $52(36.8 \%)$ & 88 (30.9\%) & 0.256 \\
\hline Diabetes & $63(14.8 \%)$ & $28(19.9 \%)$ & $35(12.3 \%)$ & 0.074 \\
\hline Atrial fibrillation & $37(8.7 \%)$ & $21(14.9 \%)$ & $16(5.6 \%)$ & 0.002 \\
\hline Coronary Heart Disease & $36(8.5 \%)$ & $25(17.7 \%)$ & $11(3.9 \%)$ & $<0.0001$ \\
\hline Obesity & $26(6.1 \%)$ & $10(7.1 \%)$ & $16(5.6 \%)$ & 0.636 \\
\hline Chronic kidney disease & $25(5.9 \%)$ & $16(11.3 \%)$ & $9(3.2 \%)$ & 0.0007 \\
\hline Chronic heart failure & $21(4.9 \%)$ & $12(8.5 \%)$ & $9(3.2 \%)$ & 0.027 \\
\hline Chronic Liver disease & $21(4.9 \%)$ & $11(7.8 \%)$ & $10(3.5 \%)$ & 0.085 \\
\hline Chronic obstructive lung disease & $20(4.7 \%)$ & $9(6.4 \%)$ & $11(3.9 \%)$ & 0.342 \\
\hline History of malignancy & $18(4.2 \%)$ & $4(2.8 \%)$ & $14(4.9 \%)$ & 0.467 \\
\hline Active malignancy & $16(3.8 \%)$ & $8(5.7 \%)$ & $8(2.8 \%)$ & 0.182 \\
\hline Dementia & $12(2.8 \%)$ & $9(6.3 \%)$ & $3(1.1 \%)$ & 0.005 \\
\hline Charlson comorbidity index & $3(1-4)$ & $4(3-5)$ & $2(1-3)$ & $<0.0001$ \\
\hline Number of comorbidities & & & & $<0.0001$ \\
\hline 0 & $155(36.3 \%)$ & $25(17.7 \%)$ & $130(45.6 \%)$ & \\
\hline 1 & $145(34.0 \%)$ & $52(36.9 \%)$ & $93(32.6 \%)$ & \\
\hline 2 & $73(17.1 \%)$ & $34(24.1 \%)$ & $39(13.7 \%)$ & \\
\hline
\end{tabular}




\begin{tabular}{|c|c|c|c|c|}
\hline $\begin{array}{l}\text { Median time from symptoms onset to } \\
\text { hospitalization }\end{array}$ & $7(3-10)$ & $6(3-8)$ & $8(4-11)$ & 0.037 \\
\hline Time of hospital admission & & & & 0.025 \\
\hline From February, 21 to March, 3 & $137(32.2 \%)$ & $36(25.5 \%)$ & $101(35.4 \%)$ & \\
\hline From March, 4 to March, 16 & $165(38.7 \%)$ & $67(40.6 \%)$ & $98(34.3 \%)$ & \\
\hline From March, 17 to March, 30 & $124(29.1 \%)$ & $38(27.0 \%)$ & $86(30.2 \%)$ & \\
\hline Glutamic oxaloacetic transaminase, U/L & $41(28-64)$ & $44(29-70)$ & $40(27-57)$ & 0.117 \\
\hline Glutamic pyruvic transaminase, $\mathrm{U} / \mathrm{L}$ & $32(21-48)$ & $34(23-53)$ & $31(21-44)$ & 0.258 \\
\hline C-reactive protein, $\mathrm{mg} / \mathrm{dL}$ & $\begin{array}{l}8.23(4.14- \\
14.75)\end{array}$ & $\begin{array}{l}10.40(5.85- \\
15.00)\end{array}$ & $\begin{array}{l}7.64(3.62- \\
14.54)\end{array}$ & 0.008 \\
\hline C-reactive protein $>10 \mathrm{mg} / \mathrm{dL}$ & $188(44.1 \%)$ & $83(58.9 \%)$ & $105(36.8 \%)$ & $<0.0001$ \\
\hline Creatinine, $\mathrm{mg} / \mathrm{dL}$ & $\begin{array}{l}0.89(0.72- \\
1.11)\end{array}$ & $\begin{array}{l}0.90(0.75- \\
1.16)\end{array}$ & $\begin{array}{l}0.87(0.71- \\
1.09)\end{array}$ & 0.132 \\
\hline Lactate dehydrogenase, $\mathrm{U} / \mathrm{L}$ & $\begin{array}{l}365(304- \\
446)\end{array}$ & $380(325-455)$ & $\begin{array}{l}365(294- \\
446)\end{array}$ & 0.075 \\
\hline Lactate dehydrogenase $>245 \mathrm{U} / \mathrm{L}$ & $369(86.6 \%)$ & $129(91.5 \%)$ & $240(84.2 \%)$ & 0.054 \\
\hline Troponine, ng/L & $26(10-108)$ & $21(10-55)$ & $37(11-119)$ & 0.103 \\
\hline White Cell Blood Count, $\times 10 \rrbracket$ per L & $\begin{array}{l}6.73(5.18- \\
9.15)\end{array}$ & $\begin{array}{l}7.02(4.95- \\
8.90)\end{array}$ & $\begin{array}{l}6.65(5.35- \\
9.3)\end{array}$ & 0.423 \\
\hline Lymphocyte Count, $\times 10 \bigotimes$ per L & $\begin{array}{l}0.80(0.60- \\
1.00)\end{array}$ & $\begin{array}{l}0.74(0.60- \\
0.97)\end{array}$ & $\begin{array}{l}0.80(0.60- \\
1.01)\end{array}$ & 0.087 \\
\hline Lymphocyte Count $<1.5 \times 10 \otimes$ per L & $398(93.3 \%)$ & $135(95.7 \%)$ & $263(92.3 \%)$ & 0.250 \\
\hline Neutrophil Count, $\times 10 \rrbracket$ per L & $\begin{array}{l}5.27(3.9- \\
7.72)\end{array}$ & $\begin{array}{l}5.50(3.61- \\
7.68)\end{array}$ & $\begin{array}{l}5.2(3.94- \\
7.75)\end{array}$ & 0.455 \\
\hline Platelet Count, $\times 10 \otimes$ per $\mathrm{L}$ & $\begin{array}{l}204(152- \\
287)\end{array}$ & $201(144-263)$ & $\begin{array}{l}207(154- \\
296)\end{array}$ & 0.184 \\
\hline Platelet Count $<150 \times 10 \rrbracket$ per $\mathrm{L}$ & $100(23.5 \%)$ & $39(27.7 \%)$ & $61(21.4 \%)$ & 0.236 \\
\hline Pneumonia at chest $\mathrm{X}$-ray & 301 (70.7\%) & $130(92.2 \%)$ & $171(60.0 \%)$ & $<0.0001$ \\
\hline
\end{tabular}

Data are expressed as median (interquartile range) or $\mathrm{n}(\%)$.

Table 2. Treatments and outcomes of patients. 


\begin{tabular}{|ll|}
\hline & $\mathrm{N}=426$ \\
\hline Treatments & \\
\hline Lopinavir /ritonavir & $174 / 354(49.1 \%)$ \\
\hline Hydroxychloroquine & $249 / 354(70.3 \%)$ \\
\hline Corticosteroids & $70 / 349(20.0 \%)$ \\
\hline Antibiotics & $304 / 358(84.9 \%)$ \\
\hline Tocilizumab & $22(5.2 \%)$ \\
\hline Outcomes & \\
\hline Death & $141(33.1 \%)$ \\
\hline Median time from symptoms onset to death (days) & $11(3-19)$ \\
\hline Median time from hospitalization to death (days) & $6(3-11)$ \\
\hline Admission to ICU & $41(9.6 \%)$ \\
\hline Median time from symptoms onset to ICU admission (days) & $11(8-13)$ \\
\hline Median time from hospitalization to ICU admission (days) & $4(2-6)$ \\
\hline Discharge & $239(56.1 \%)$ \\
\hline Median time from symptoms onset to discharge (days) & $19(9-24)$ \\
\hline Median time from hospitalization to discharge (days) & $10(5-16)$ \\
\hline Respiratory Failure & $245(57.5 \%)$ \\
\hline Acute kidney injury & $26(6.1 \%)$ \\
\hline Acute cardiac injury & $14(3.3 \%)$ \\
\hline Septic Shock & $7(1.6 \%)$ \\
\hline Thromboembolic events & $7(1.6 \%)$ \\
\hline
\end{tabular}

Data are expressed as median (interquartile range) or $\mathrm{n}(\%)$.

Table 3. Competing risk analysis by Fine and Gray model for in-hospital mortality and discharge. 


\begin{tabular}{|c|c|c|c|c|c|c|c|c|}
\hline & \multicolumn{8}{|c|}{ Fine and Grey model } \\
\hline & \multicolumn{4}{|c|}{ In-hospital mortality } & \multicolumn{4}{|c|}{ Discharge } \\
\hline & Beta & $\begin{array}{l}\text { Standard } \\
\text { Error }\end{array}$ & $\begin{array}{l}\text { Hazard } \\
\text { ratio }\end{array}$ & $\begin{array}{l}\mathrm{p} \\
\text { value }\end{array}$ & Beta & $\begin{array}{l}\text { Standard } \\
\text { Error }\end{array}$ & $\begin{array}{l}\text { Hazard } \\
\text { Ratio }\end{array}$ & $p$ value \\
\hline \multirow[t]{2}{*}{ Age $70-79$ years } & 1.49 & 0.27 & 4.42 & $<0.000$ & -0.95 & 0.19 & 0.39 & $<0.0001$ \\
\hline & & & & 1 & & & & \\
\hline \multirow[t]{2}{*}{ Age $>80$ years } & 2.05 & 0.29 & 7.75 & $<0.000$ & -1.31 & 0.25 & 0.27 & $<0.0001$ \\
\hline & & & & 1 & & & & \\
\hline Male sex & 0.64 & 0.22 & 1.89 & 0.003 & -0.66 & 0.14 & 0.52 & $<0.0001$ \\
\hline $\begin{array}{l}\text { Number of } \\
\text { comorbidities }\end{array}$ & 0.52 & 0.25 & $3.63^{\star}$ & 0.038 & -0.62 & 0.21 & $0.08^{\star *}$ & 0.003 \\
\hline $\begin{array}{l}\text { Admission between } \\
\text { March, } 4 \text { and } \\
\text { March, } 16\end{array}$ & 0.84 & 0.23 & 2.32 & 0.001 & -0.42 & 0.17 & 0.66 & 0.015 \\
\hline $\begin{array}{l}\text { Admission between } \\
\text { March, } 17 \text { and } \\
\text { March, } 30\end{array}$ & 0.52 & 0.25 & 1.68 & 0.048 & -0.39 & 0.16 & 0.68 & 0.017 \\
\hline $\begin{array}{l}\text { Lymphocyte Count, } \\
\times\end{array}$ & -0.22 & 0.22 & 0.80 & 0.316 & 0.12 & 0.03 & 1.13 & 0.0001 \\
\hline \multicolumn{9}{|l|}{ 10ه per L } \\
\hline Hydroxychloroquine & -0.13 & 0.27 & 0.88 & 0.639 & -0.27 & 0.16 & 0.76 & 0.760 \\
\hline No ICU admission & 0.34 & 0.45 & 1.41 & 0.449 & \multicolumn{4}{|c|}{ Baseline } \\
\hline $\begin{array}{l}\text { Time to ICU } \\
\text { admission lower } \\
\text { than } 3 \text { days }\end{array}$ & \multicolumn{4}{|c|}{ Baseline } & -0.61 & 0.32 & 0.54 & 0.056 \\
\hline $\begin{array}{l}\text { Time to ICU } \\
\text { admission between } \\
4 \text { and } 6 \text { days }\end{array}$ & -0.61 & 0.63 & 0.54 & 0.330 & -0.02 & 0.27 & 0.98 & 0.955 \\
\hline $\begin{array}{l}\text { Time to ICU } \\
\text { admission }>7 \text { days }\end{array}$ & -1.67 & 0.65 & 0.19 & 0.010 & -0.01 & 0.01 & 0.99 & 0.996 \\
\hline
\end{tabular}

*Three comorbidities or more versus no comorbidities. Hazard ratio was 2.36 for two comorbidities versus no comorbidities and 1.54 for one comorbidity versus no comorbidity.

** Three comorbidities or more versus no comorbidities. Hazard ratio was 0.19 for two comorbidities versus no comorbidities and 0.44 for one comorbidity versus no comorbidity.

\section{Figures}




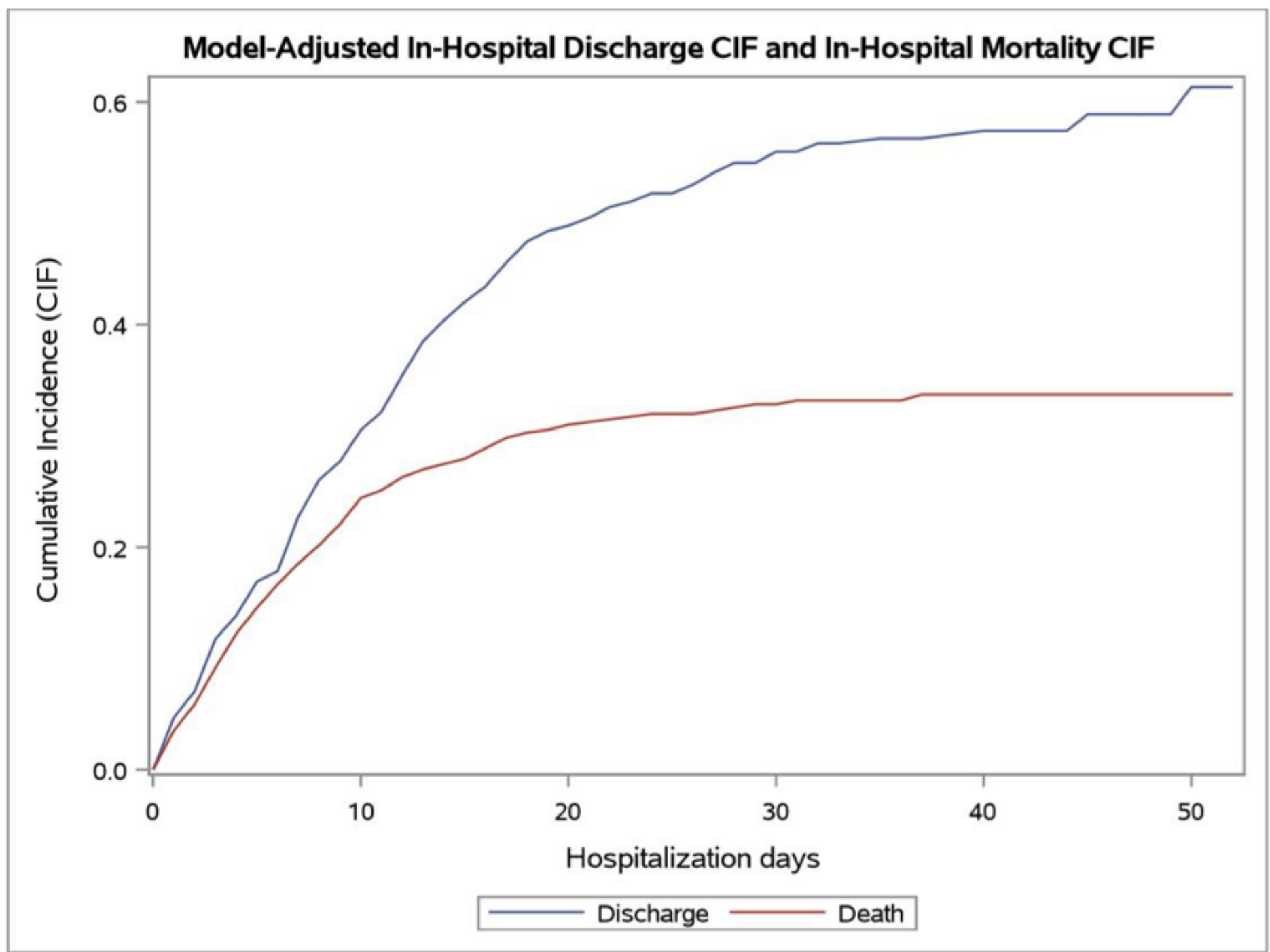

Figure 1

Cumulative incidence functions for in-hospital mortality and discharge of patients with Coronavirus Disease19. 

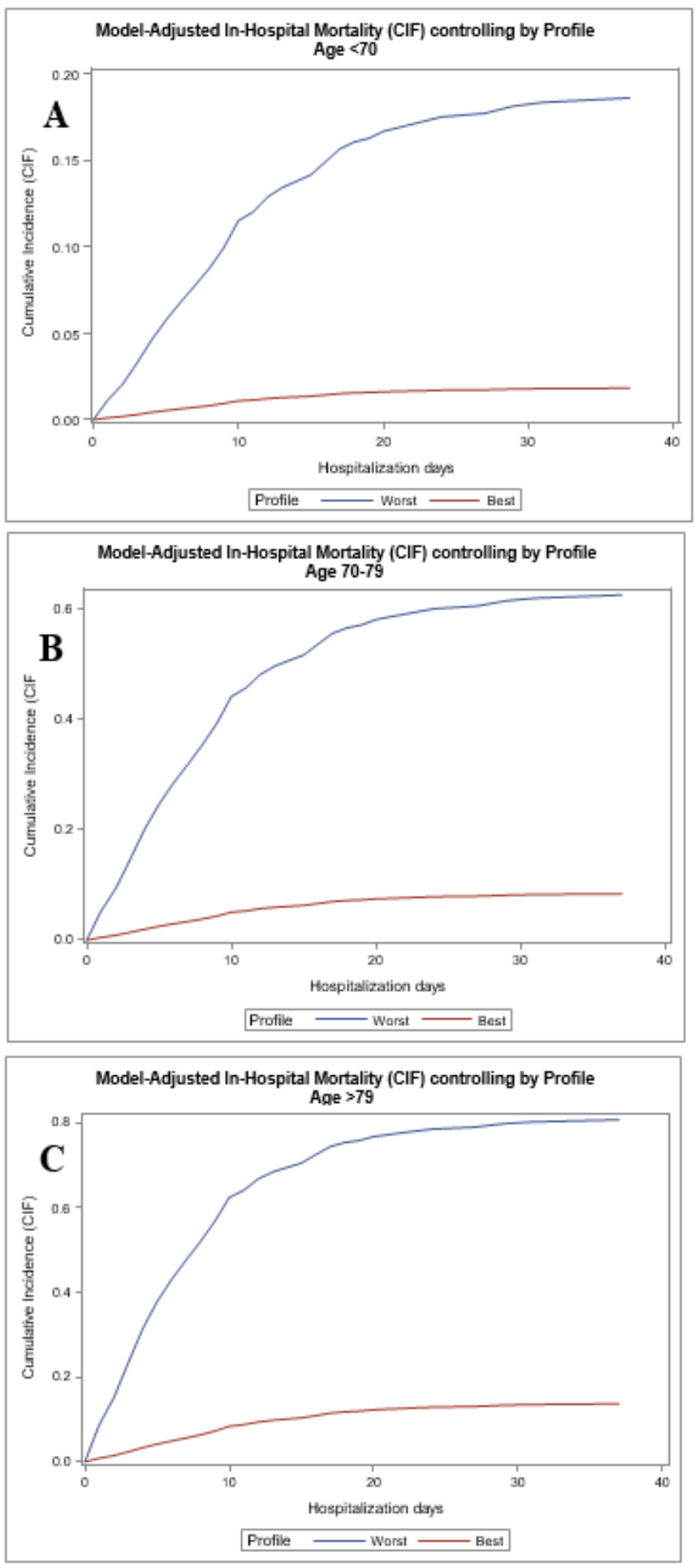

\section{Figure 2}

Cumulative incidence functions for in-hospital mortality performed using the parameter estimates of the Fine and Gray model and considering the best patient profile (female sex, number of comorbidities lower than 3 admitted between 21 February to 3 March 2020) and the worst patient profile (male sex, number of comorbidities higher than 3, admitted between 4 March and 16 March 2020) according to age groups. A: age lower than 70 years. B: age between 70 and 79 years. C: age higher than 79 years. 


\section{Supplementary Files}

This is a list of supplementary files associated with this preprint. Click to download.

- SupplementalSRManuscriptVZstatementmerged.pdf 